\title{
Determination of Slow-Neutron Fluence Rate Using Gamma-Ray Spectroscopy
}

\author{
B. GaWeŁCZYK And A. KOnefat* \\ Institute of Physics, University of Silesia in Katowice, Katowice, Poland \\ Doi: 10.12693/APhysPolA.139.280 \\ *e-mail: adam.konefal@us.edu.pl
}

\begin{abstract}
In this work, the method of determination of the slow-neutron fluence rate using a gamma-ray spectroscopy with a high-purity germanium detector (HPGe) and Monte Carlo calculations was presented. The prompt gamma rays with energies of 595.9, 867.9, and $1204.2 \mathrm{keV}$, originating from the nuclear reaction ${ }^{73} \mathrm{Ge}(n, \gamma){ }^{74} \mathrm{Ge}$ in the germanium crystal of the HPGe detector, were registered. A special system composed of two neutron sources (Cf-252, PuBe), a lead shield and a HPGe detector was made to find correlations between net areas of the peaks from these prompt gamma rays in the measured spectra and the fluence rate of slow neutrons getting to the germanium crystal. The net areas of the peaks were related to the slow-neutron fluence rate using the Monte Carlo calculations based on the GEANT4 code. The presented method using the gamma-ray spectroscopy can be applied in laboratories which do not have detectors for neutron measurements.
\end{abstract}

topics: neutrons, HPGe, Monte Carlo

\section{Introduction}

Technological progress leads to the prevalence of devices using radioactive sources or producing ionization radiation. Various types of radiation are commonly applied in industry, in radiotherapy and nuclear medicine, as well as in many scientific laboratories. The use of ionization radiation requires a parallel application of specialist equipment suitable for measurements of this radiation. Typical equipment is usually limited to gamma, beta, and possibly alpha radiation. The measurement of neutrons often poses a problem since the majority of laboratories do not have detectors for neutron measurement.

However, neutrons are often a secondary radiation unwanted in industrial, medical and scientific activities [1-6]. Neutrons can induce nuclear reactions with a production of many radioisotopes $[7,8]$, leading to radiation damages in microelectronics which often translates into financial losses. This problem is particularly important for oncological patients with an implanted cardiac device when treated by high-energy photon radiotherapy. Any damages to such devices due to radiation may cause their malfunctioning $[9,10]$ and, consequently, pose a serious threat to patients' lives. Moreover, the neutrons can burden medical workers and patients with an additional dose, also from radiation emitted by the induced radioisotopes [11, 12].

Therefore, a validation of a method enabling the determination of neutron fluence rate or at least the identification of the neutron field is a significant challenge. The present work's goal was to respond to this challenge. Our purpose was to present a method of determination of the slow-neutron fluence rate (from thermal energies up to $1 \mathrm{eV}$ ), basing on a gamma-ray spectroscopy using a HPGe and Monte Carlo calculations.

\section{Materials and methods}

The used method was based on a gamma-ray spectroscopy to measure prompt $\gamma$ rays with energies of 595.9, 867.9, and $1204.2 \mathrm{keV}$, originating from the nuclear reaction ${ }^{73} \mathrm{Ge}(n, \gamma){ }^{74} \mathrm{Ge}$ induced by neutrons in the germanium crystal of a HPGe detector. This reaction is characterized by a relatively high thermal neutron cross-section of $14.4 \mathrm{~b}$ and by three resonances of over $1000 \mathrm{~b}$ and several ones of over $100 \mathrm{~b}$ in the energy range from $0.1 \mathrm{keV}$ to $1 \mathrm{eV}$ [13]. Appearance of the peaks at the three mentioned energies in the gamma-ray spectrum confirms a presence of a slow-neutron field in a measuring location.

A special set composed of a HPGe detector and an InSpector1000 detection system by Canberra, a lead shield to reduce a photon background in the measuring location and two neutron sources $\left({ }^{252} \mathrm{Cf}\right.$, $\mathrm{Pu}-\mathrm{Be}$ ) inside the shielding containers (see Fig. 1) were used in the experimental part of this work. The InSpector1000 detection system consisting of the $\mathrm{NaI}(\mathrm{Tl})$ scintillation detector and the $8 \mathrm{~cm}$ long helium chamber with a gas pressure of $2 \mathrm{~atm}$ was applied for the test of effectiveness of the lead shield. The HPGe detector was connected to a multichannel analyzer installed in a laptop and operated by the Genie 2000 software. 


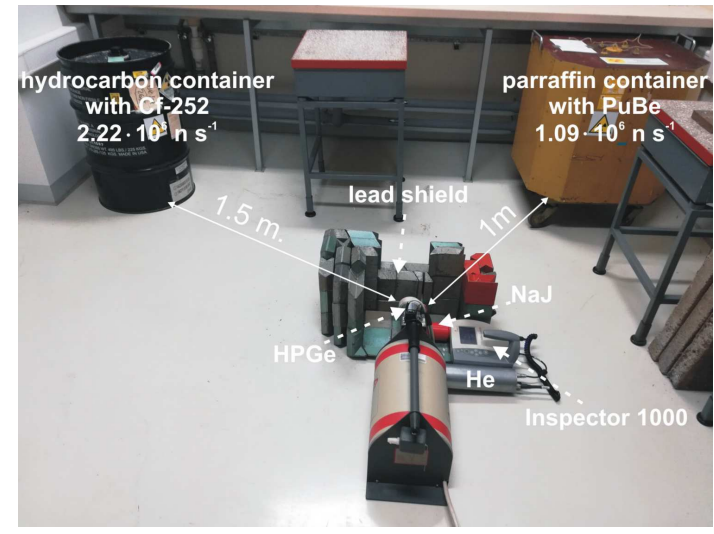

Fig. 1. View of the used experimental setup.

The net area of the peaks at 595.9, 867.9, and $1204.2 \mathrm{keV}$ can be related to the fluence rate of slow neutrons in the measuring location. The dependence between the measured number of counts in the net area of the peak per second $\mathrm{d} N / \mathrm{d} t$ and the slow-neutron fluence rate $\mathrm{d} \phi / \mathrm{d} t$ was obtained using the Monte Carlo calculations. It can be determined by the correlation factor $C$ linking these two quantities. Namely,

$$
C=\frac{\mathrm{d} \phi / \mathrm{d} t}{\mathrm{~d} N / \mathrm{d} t}
$$

The energy spectrum of slow neutrons always has the same shape. The slow-neutron energy is distributed according to the Maxwell-Boltzmann distribution law. Therefore, it is easy to separate the slow-neutron contribution from the full spectrum and to define $\phi$ as the sum of all slow neutrons reaching the germanium crystal per unit time, normalized to a surface unit. It can be written accordingly

$$
\frac{\mathrm{d} \phi}{\mathrm{d} t}=\int_{0}^{1 \mathrm{eV}} \frac{\mathrm{d} \phi(E)}{\mathrm{d} t} \mathrm{~d} E,
$$

where $\mathrm{d} \phi(E) / \mathrm{d} t$ is the fluence rate of slow neutrons with energy $E$. The integration limits are determined by the energy range in which the reaction ${ }^{73} \mathrm{Ge}(n, \gamma){ }^{74} \mathrm{Ge}$ occurs. The Monte Carlo calculations based on the GEANT4 code with high precision models of neutron interactions using the ENDF cross-sections were applied to determine the slowneutron fluence rate in the measuring location for the applied experimental setup.

The so determined factor $C$ can be used for measurements of the slow-neutron fluence rate in another location where an unknown slow-neutron field occurs. Then, the following relationship is used:

$$
\frac{\mathrm{d} \phi^{\prime}}{\mathrm{d} t}=C \frac{\mathrm{d} N^{\prime}}{\mathrm{d} t},
$$

where $\mathrm{d} \phi^{\prime} / \mathrm{d} t$ and $\mathrm{d} N^{\prime} / \mathrm{d} t$ are, respectively, the slow-neutron fluence rate and the measured number of counts in the net area of the peak at 595.9, 867.9 , or $1204.2 \mathrm{keV}$ per second in any measurement location.

\section{Results}

A series of measurements of gamma-ray spectra were made in the vicinity of two neutron sources ${ }^{252} \mathrm{Cf}$ and $\mathrm{Pu}-\mathrm{Be}$, as shown in Fig. 1. The representative spectrum for the experimental session is shown in Fig. 2. This spectrum was divided into energy ranges important for the presented method. The net areas for the selected peaks (see Table I) were estimated with the Genie 2000 program used for controlling the germanium detector.

The spectrum of slow neutrons calculated using the Monte Carlo Geant4 code [14] is presented in Fig. 3. The domination of neutrons from ${ }^{252} \mathrm{Cf}$ is visible in the measuring location. The determined values of $\mathrm{d} \phi / \mathrm{d} t$ in the germanium crystal of the used HPGe detector were $4.8 \mathrm{~cm}^{-2} \mathrm{~s}^{-1}$ and $0.8 \mathrm{~cm}^{-2} \mathrm{~s}^{-1}$ for the ${ }^{252} \mathrm{Cf}$ and $\mathrm{Pu}-\mathrm{Be}$ sources, respectively.

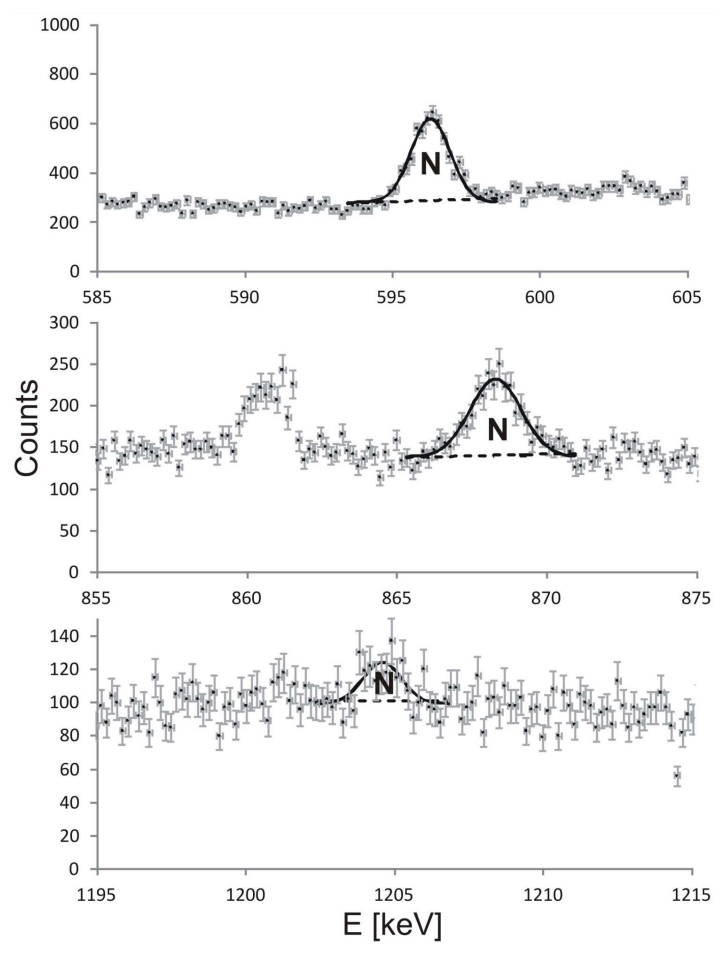

Fig. 2. Fragments of the gamma-ray spectrum with visible peaks at $595.9,867.9$, and $1204.2 \mathrm{keV}$. In order to calculate the net areas $N$ for the selected peaks, a Gaussian fit was used after the background cut-off. The time of the measurements was $30 \mathrm{~h}$.

TABLE I

Net areas for the selected peaks related to $1 \mathrm{~s}$ and values of the correlation factor $C$ for the used HPGe detector.

\begin{tabular}{c|c|c}
\hline \hline Energy $[\mathrm{keV}]$ & $\mathrm{d} N / \mathrm{d} t\left[\mathrm{~s}^{-1}\right]$ & $C\left[\mathrm{~cm}^{-2}\right]$ \\
\hline 595.9 & $0.0273 \pm 0.0014$ & $205 \pm 11$ \\
867.9 & $0.0104 \pm 0.0010$ & $539 \pm 53$ \\
1204.2 & $0.0020 \pm 0.0015$ & $2774 \pm 2050$
\end{tabular}




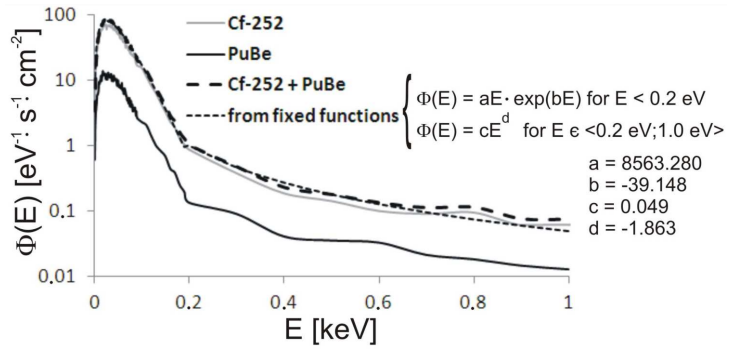

Fig. 3. Spectra of neutrons getting to the germanium crystal of the HPGe detector per second in the measuring location (see also Fig. 1). Contributions from the sources ${ }^{252} \mathrm{Cf}$ and $\mathrm{Pu}$-Be as well as the total spectrum and the spectrum estimated from the fitted function used for the calculation of $\mathrm{d} \phi / \mathrm{d} t($ see $(2))$ are presented.

The factor $C$, importantly, is characteristic of each HPGe detector. In addition, it depends on the detector efficiency, i.e., $C$ does not change only if the absolute efficiency of the detector is constant.

\section{Discussion and conclusions}

All the three peaks from the reaction ${ }^{73} \mathrm{Ge}(n, \gamma){ }^{74} \mathrm{Ge}$ were visible in the measured spectra. However, only the peaks at $595.9 \mathrm{keV}$ and at $867.9 \mathrm{keV}$ are recommended to be used because of the acceptable uncertainty of their net areas which translates into the accuracy of the determination of the slow-neutron fluence rate. The presented method was prepared for the slow-neutron field characterized by $\mathrm{d} \phi / \mathrm{d} t=5.6 \mathrm{~cm}^{-2} \mathrm{~s}^{-1}$. This proves that the developed method can be used for weak slow-neutron fields, which, however, is related to the relatively long measurement time of the gamma-ray spectrum.

The undoubted advantage of the presented method is the fact that the HPGe detector is now often in the possession of radiation therapy centers with possibilities of carrying out advanced numerical calculations. The limitation of the method's application, however, is the required low level of gamma radiation background. Therefore, in the vicinity of strong gamma radiation sources, the detector has to be shielded with a layer of lead or an equivalent shielding material. The problem may also be access to a neutron source needed to determine the correlation factor $C$.

\section{References}

[1] A. Konefał, M. Dybek, W. Zipper, W. Eobodziec, K. Szczucka, Nukleonika 50, 73 (2005).

[2] A. Konefał, A. Orlef, M. Dybek, Z. Maniakowski, K. Polaczek-Grelik, W. Zipper, Phys. Med. 24, 212 (2008).

[3] H.R. Vega-Carrillo, A. Baltazar-Raigosa, J. Radiat. Nucl. Chem. 287, 323 (2011).

[4] M. Janiszewska, K. Polaczek-Grelik, M. Raczkowski, B. Szafron, A. Konefał, W. Zipper, Strahlenther Onkol. 190, 459 (2014).

[5] A. Konefał, M. Łaciak, A. Dawidowska, W. Osewski, Radiat. Protect. Dosim. 162, 197 (2014).

[6] M. Bieniasiewicz, A. Konefał, J. Wendykier, A. Orlef, Acta Phys. Pol. B 47, 771 (2016).

[7] A. Konefał, K. Polaczek-Grelik, W. Zipper, Radiat. Protect. Dosim. 128, 133 (2008).

[8] A. Konefał, A. Orlef, M. Bieniasiewicz, $R a$ diat. Meas. 86, 8 (2016).

[9] D. Bisello, A. Candeloria, N. Dzysiuk, J. Esposito, P. Mastinu, S. Mattiazzo, G. Prete, L. Silvestrin, J. Wyss, Phys. Proced. 26, 284 (2012).

[10] A. Konefał, S. Blamek, A. Wrońska, A. Orlef, M. Sokół, M. Tajstra, M. Gąsiorek, Appl. Radiat. Isot. 163, 109206 (2020).

[11] K. Polaczek-Grelik, A. Orlef, M. Dybek, A. Konefał, W. Zipper, Appl. Radiat. Isot. 68, 763 (2010).

[12] K. Polaczek-Grelik, B. Karaczyn, A. Konefał, Appl. Radiat. Isot. 70, 2332 (2012).

[13] National Nuclear Data Center, Evaluated Nuclear Data File.

[14] CERN, GEANT4 project site. 